OPEN ACCESS

Edited by:

Ho-Chang Kuo,

Kaohsiung Chang Gung Memorial

Hospital, Taiwan

Reviewed by:

Sung-Chou Li,

Kaohsiung Chang Gung Memorial

Hospital, Taiwan

Akihiro Nakamura,

Kyoto Prefectural University of

Medicine, Japan

*Correspondence:

Yu Jiang

jy19770101@163.com

†These authors have contributed equally to this work

Specialty section

This article was submitted to

Pediatric Immunology,

a section of the journal

Frontiers in Pediatrics

Received: 01 November 2021

Accepted: 17 January 2022

Published: 11 February 2022

Citation:

Tian F, Ma L, Zhao R, Ji L, Wang X

Sun $W$ and Jiang $Y$ (2022) Correlation Between Matrix Metalloproteinases With Coronary Artery Lesion Caused

by Kawasaki Disease.

Front. Pediatr. 10:802217.

doi: 10.3389/fped.2022.802217

\section{Correlation Between Matrix Metalloproteinases With Coronary Artery Lesion Caused by Kawasaki Disease}

\author{
Fang Tian 1,2†, Linxi Ma ${ }^{3+}$, Renbing Zhao ${ }^{2}$, Lijuan $\mathrm{Ji}^{2}$, Xiufen Wang ${ }^{2}$, Wenli Sun ${ }^{2}$ and \\ Yu Jiang ${ }^{2 \star}$ \\ ${ }^{1}$ Heart Center of Women and Children Hospital of Qingdao University, Qingdao, China, ${ }^{2}$ Department of Pediatric, Maternal \\ and Child Health Hospital, Zibo, China, ${ }^{3}$ Nanchang University Queen Mary School, Nanchang, China
}

This study was designed to clarify the role of matrix metalloproteinases (MMPs) in coronary artery lesions (CAL). Serum samples were acquired from healthy, febrile, and Kawasaki disease (KD) children with or without CAL. Standard blood parameters were examined and enzyme-linked immunosorbent assay (ELISA) was used to assess the levels of MMP-2 and MMP-9. Intravenous immunoglobulin (IVIG) therapy was conducted on the KD patients and the changes of MMPs before and after treatment were compared. The correlations between MMP levels and clinical parameters were also evaluated. Compared to febrile and healthy controls, KD patients demonstrated clinical signs characteristic of abnormal immunoregulation. However, the clinical parameters of KD patients with or without CAL were not significantly different. MMP-2 and MMP-9 levels, however, were significantly higher in KD patients with CAL than those without CAL. IVIG treatment effectively downregulated the levels of MMPs in KD patients, which was more prominent in those with CAL. Significant correlations were found between MMP levels and some clinical parameters of KD, such as fever time, white blood cell count, etc. The upregulation of MMPs significantly correlates with coronary artery aneurysms (CAAs) in $\mathrm{KD}$ patients, making it important biomarkers of CAL in KD patients.

Keywords: Kawasaki disease, coronary artery lesions, matrix metalloproteinases, IVIG therapy, KD patients

\section{INTRODUCTION}

Kawasaki disease (KD), also known as cutaneous mucosal lymph node syndrome, is a systemic vasculitis syndrome caused by an autoimmune disorder (1). KD is characterized by activation of the immune system and extensive damage to the endothelial system (2). During the acute phase of $\mathrm{KD}$, the pronounced activation of immune system results in production of inflammatory mediators and proteases, as well as reactive oxygen species, which are believed to induce lesions in the vascular systems (3). Infants and young children with KD are extremely vulnerable to coronary artery lesions (CALs), including myocardial infarctions, coronary artery fistula formations, coronary artery dilatations, and coronary artery aneurysms (CAAs). Despite progresses in clinical management of $\mathrm{KD}$, unfortunately $20 \%$ of children with KD progress to CAL and have a high mortality rate (4).

Matrix metalloproteinases (MMPs) are a family of enzymes responsible for degradation of extracellular matrix (ECM). The link of overexpression of specific MMPs and a number of human 
malignancies have been established (5). For example, the high expression of MMPs are found to be correlated with periodontal disease (6), atherosclerosis (7), cancer (8) and rheumatoid arthritis (9). MMPs are important molecules in regulating the structure and function of the coronary artery wall, therefore making them essential biomarkers and therapeutic targets for the treatment of vascular diseases (10). MMP-9 and MMP7 have been reported to be involved in CAA (11). The proinflammatory factor TNF- $\alpha$ can induce the production of MMP9 (12), which consequently contributes to the progression of vascular injury in KD. However, not all MMPs are effective diagnostic markers and treatment targets for CAL caused by $\mathrm{KD}$ and further evidences are in need to support the use of certain MMPs for accurate diagnosing and treating CAL caused by KD. Previously, MMP-2 and MMP-9 have been the focus of earlier $\mathrm{KD}$ research $(13,14)$, despite that the association of these two MMPs with CAL in KD remains unclear. In this study, we strived to analyze the relationship between MMP-2/MMP-9 and CALs in KD. By measuring serum levels of different MMPs, including MMP-2 and MMP-9, we aim to identify correlations between MMP expression and traditional clinical parameters of KD. Our findings could potentially clarify the role of MMP-2 and MMP-9 in KD, particularly those with CAL, and facilitate the development of new and effective treatment for KD.

\section{METHODS}

\section{Patients}

The study was conducted in accordance with the Declaration of Helsinki (as revised in 2013). The study was approved by the ethics committee of Women and Children's Hospital of Qingdao University (2010071). Informed consent was taken from all the patients' parents. KD children who were admitted at Women and Children's Hospital of Qingdao University from December 2010 to July 2019 were included in the study. Diagnostic of KD was confirmed according to the criteria by the Japan Kawasaki Disease Research Committee. Exclusion criteria included: no hormones, immunosuppressants, and gamma globulin have been used in the past 2 weeks; cardiopulmonary and basic diseases; incomplete clinical data, or history of hospital transfer during treatment; unwilling to cooperate with the treatment.

\section{Diagnosis for Coronary Artery Dilation}

The following criteria were used for diagnosis of coronary artery lesions (1): normal: $Z$ value $<2$; (2) only dilation: $Z$ value 2 to $<2.5$; or initial $Z$ value $<2, Z$ value decline during follow-up (usually $6-12$ months) $\geq 1$; (3) small Coronary aneurysm: $Z$ value $\geq 2.5 \sim<5$; (4) Medium-sized coronary aneurysm: $Z$ value $\geq 5$ $\sim<10$, and absolute value of inner diameter $<8 \mathrm{~mm}$; (5) Giant coronary aneurysm: $Z$ value $\geq 10$, or inner diameter. Patients with normal coronary artery (CAL-) were defined as those with $\mathrm{Z}$-score $<2.0$, while those with $\mathrm{Z}$-score $\geq 2.0$ were considered to be CAL+ (15).

\section{Specimen Acquisition}

On the 2nd and 10th day after admission, $4 \mathrm{ml}$ of peripheral venous blood was withdrawn from 140 cases of $\mathrm{KD}$ children after early morning fasting. Blood sample was centrifuged at $3,000 \mathrm{r} / \mathrm{min}$ for $30 \mathrm{~min}$. The supernatant was collected and placed in PC Store in the tube at $-75^{\circ} \mathrm{C}$. Blood sample from thirty healthy children in the normal control group were blood drawn, centrifuged, and frozen in the same method.

\section{Statistical Analysis}

Data sorting, screening and statistical analysis were performed with SPSS18.0 software: Differences between groups were compared using the Mann-Whitney $U$ test, and subgroup analysis was performed using the Wilcoxon test. One-way ANOVA followed by Tukey's tests was applied for multiple-group comparison. Correlations analysis between MMP-2 and MMP9 levels and clinical parameters in KD patients were performed using Pearson's correlation analysis. $P<0.05$ was considered statistically significant.

\section{RESULTS}

\section{Study Enrollment and Cohort Characteristics}

One hundred five patients with KD were initially enrolled, and eventually 95 subjects completed the study and were included for analyses. Inpatients in the Heart Center of Qingdao Women and Children hospital were analyzed for demographic and clinical parameters (Table 1). The ages of patients with $\mathrm{KD}$, febrile controls (FC) and healthy controls (NC) in this study were 2.39 $\pm 1.65,2.64 \pm 1.97$, and $2.27 \pm 2.03$ years old, respectively $(p$ $=0.307)$. All groups enrolled slightly more females than males (male/female: 50/55, 25/28, and 33/39, $p=0.205$ ). Compared to the healthy control group, children in the $\mathrm{KD}$ group and $\mathrm{FC}$ group demonstrated significantly longer fever time, and higher white blood cell count (WBC), red blood cell count (RBC), hemoglobin, platelet, procalcitonin (PCT), c-reactive protein (CRP), erythrocyte sedimentation rate (ESR), interleukin-6 (IL6) and monocyte chemoattractant protein-1 (MCP-1) levels, which are signs of abnormal immunoregulation and consequence of irregular temperature regulation. These levels were even higher in the KD group than those in the FC group.

\section{Clinical Parameters of KD Patients With or Without CAL}

We next performed analysis of clinical parameters within the $\mathrm{KD}$ group and strive to identify differences between those with CAL and those without CAL. We found that fever time, WBC, $\mathrm{RBC}$, hemoglobin, platelet and PCT levels were not significantly different between the two groups but the levels of CRP, ESR, IL-6 and MCP-1 of those with CAL were markedly higher than those without CAL $(p=0.017,0.037,0.007$, and 0.012 , respectively, Table 2 ).

\section{Analysis of MMP-2, MMP-9 Levels}

We next analyzed MMP levels of patients in different groups. As shown in Table 3, MMP-2 and MMP-9 all showed markedly higher expression compared to FC and NC group $(p<0.001)$. In subgroup analysis, among KD patients, those with CAL also demonstrated pronouncedly higher MMP-2 and MMP-9 levels 
TABLE 1 | Cohort characteristics.

\begin{tabular}{|c|c|c|c|c|}
\hline & $\mathrm{KD}(n=95)$ & $\mathrm{FC}(n=53)$ & $\mathrm{NC}(n=62)$ & $p$-value \\
\hline Age (y) & $2.37 \pm 1.54$ & $2.64 \pm 1.97$ & $2.27 \pm 2.03$ & 0.307 \\
\hline Sex (male/female) & $43 / 52$ & $25 / 28$ & $33 / 39$ & 0.205 \\
\hline Fever time (day) & $6.32 \pm 1.81^{\star \star \star \& \&}$ & $2.28 \pm 2.55^{\star \star}$ & $0.00 \pm 0.00$ & $<0.001$ \\
\hline WBC $\left(10^{3} / \mu \mathrm{L}\right)$ & $16.43 \pm 5.66^{\star \star \star \& \&} \&$ & $10.65 \pm 4.49^{*}$ & $8.54 \pm 2.73$ & $<0.001$ \\
\hline $\operatorname{RBC}\left(106 / \mathrm{mm}^{3}\right)$ & $3.96 \pm 0.47^{\star \star \& \&}$ & $4.37 \pm 0.46^{\star}$ & $4.88 \pm 0.61$ & $<0.001$ \\
\hline Hemoglobin (g/dL) & $9.97 \pm 1.21^{\star \&}$ & $11.15 \pm 1.31^{\star}$ & $11.71 \pm 1.08$ & $<0.001$ \\
\hline Platelet $\left(10^{3} / \mu \mathrm{L}\right)$ & $410.64 \pm 149.38^{\star \star \star \& \& \&}$ & $354.61 \pm 112.63^{\star \star}$ & $316.45 \pm 107.82$ & $<0.001$ \\
\hline PCT (ng/L) & $1.23 \pm 0.94^{\star \star \star \& \& \&}$ & $0.67 \pm 0.26^{\star \star}$ & $0.43 \pm 0.24$ & $<0.001$ \\
\hline $\mathrm{CRP}(\mathrm{mg} / \mathrm{dL})$ & $58.31 \pm 38.95^{\star \star * \& \& \&}$ & $25.36 \pm 16.84^{\star \star}$ & $9.12 \pm 6.97$ & $<0.001$ \\
\hline $\mathrm{ESR}(\mathrm{mm} / \mathrm{h})$ & $68.85 \pm 35.07^{\star \star \star \& \& \&}$ & $35.88 \pm 22.45^{\star \star *}$ & $12.25 \pm 5.37$ & $<0.001$ \\
\hline IL-6 (pg/ml) & $211.21 \pm 30.23^{\star \star \star \& \& \& \&}$ & $105.32 \pm 20.18^{\star \star \star}$ & $22.19 \pm 7.34$ & $<0.001$ \\
\hline MCP-1 (pg/ml) & $204.47 \pm 69.32^{\star \star \star \& \&}$ & $145.37 \pm 48.12^{\star \star}$ & $104.26 \pm 39.47$ & $<0.001$ \\
\hline
\end{tabular}

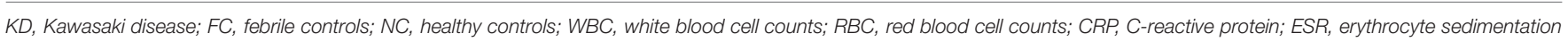
rate; PCT, procalcitonin; IL-6, interleukin 6; MCP-1, monocyte chemoattractant protein-1.

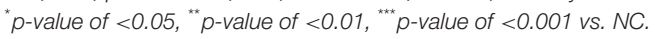

${ }^{\&} p$-value of $<0.05, \& \& p$-value of $<0.01, \& \& \& p$-value of $<0.001$ vs. $F C$.

TABLE 2 | Clinical parameters in two groups of KD patients.

\begin{tabular}{lccc}
\hline & $\begin{array}{c}\text { KD with CAL } \\
(\boldsymbol{n}=\mathbf{5 0})\end{array}$ & $\begin{array}{c}\text { KD without CAL } \\
(\boldsymbol{n}=\mathbf{4 5})\end{array}$ & p-value \\
\hline Age $(\mathrm{y})$ & $2.58 \pm 1.66$ & $2.49 \pm 1.47$ & 0.262 \\
Sex (male/female) & $24 / 26$ & $16 \pm 29$ & 0.078 \\
Fever time (day) & $6.57 \pm 2.05$ & $6.43 \pm 1.68$ & 0.446 \\
WBC $\left(10^{3} / \mu \mathrm{L}\right)$ & $16.07 \pm 6.79$ & $15.32 \pm 5.95$ & 0.587 \\
RBC $\left(106 / \mathrm{mm}^{3}\right)$ & $4.05 \pm 0.67$ & $4.06 \pm 0.53$ & 0.372 \\
Hemoglobin $(\mathrm{g} / \mathrm{dL})$ & $9.93 \pm 1.44$ & $10.07 \pm 1.52$ & 0.556 \\
Platelet $\left(10^{3} / \mu \mathrm{L}\right)$ & $410.43 \pm 147.83$ & $405.72 \pm 155.61$ & 0.401 \\
PCT $(\mathrm{ng} / \mathrm{L})$ & $1.27 \pm 0.84$ & $1.24 \pm 0.85$ & 0.207 \\
CRP $(\mathrm{mg} / \mathrm{dL})$ & $69.32 \pm 37.04$ & $47.53 \pm 25.26$ & $0.017^{\star}$ \\
ESR $(\mathrm{mm} / \mathrm{h})$ & $69.74 \pm 33.82$ & $57.23 \pm 34.03$ & $0.037^{*}$ \\
IL-6 $(\mathrm{pg} / \mathrm{ml})$ & $210.36 \pm 32.51$ & $187.45 \pm 29.48$ & $0.007^{\star *}$ \\
MCP-1 (pg/ml) & $226.58 \pm 67.35$ & $197.21 \pm 54.71$ & $0.012^{*}$ \\
\hline
\end{tabular}

$K D$, Kawasaki disease; CALs, coronary artery lesions; WBC, white blood cell counts; RBC, red blood cell counts; $C R P, C$-reactive protein; ESR, erythrocyte sedimentation rate; $P C T$, procalcitonin; IL-6, interleukin 6; MCP-1, monocyte chemoattractant protein-1.

* $p$-value of $<0.05, " p$-value of $<0.01$, comparison between two KD groups.

than those without CAL ( $p=0.014$ and 0.006 , respectively). Therefore, this data implicates that MMPs likely contribute to the pathogenesis of KD and CAL.

\section{Reduction of MMPs by IVIG Therapy}

We further analyzed the change of MMPs before and after IVIG therapy. Our data showed that after IVIG treatment, MMP-2 and MMP-9 levels of KD patients decreased 409.32 and 205.43 $\mathrm{pg} / \mathrm{mL}$, respectively, compared to pretreatment levels $(p<0.001$ for MMP-2 and MMP-9, Table 4). Further, our analyses showed that KD patients with CAL exhibited higher reduction of MMP2 and MMP-9 compared to those without CAL ( $p=0.037$ for MMP-2 and $p=0.012$ for MMP-9).

\section{Correlations Analysis Between MMP-2, MMP-9 Levels and Clinical Parameters in KD Patients}

Next, we examined the correlation of MMP-2 and MMP-9 levels with clinical parameters of KD. As shown in Table 5, significant correlations were found between MMP-2 and MMP-9 with fever time, PCT, CRP, IL-6, and MCP-1; MMP-2 and MMP-9 also showed significant correlations with WBC.

\section{DISCUSSION}

Here we report that the increased expression of MMP-2 and MMP-9 are correlated KD, particularly KD patients with CAL. The serum samples of KD patients, along with healthy patients and febrile patients as controls, were used for analysis. This approach is also adopted by another study by Takeshita et al., which showed the elevated serum levels of MMPs in KD patients. To use serum level of MMPs as biomarkers, our hypothesis was that $\mathrm{KD}$ is characterized by activated monocytes and neutrophils in circulation, which produce a large amount of soluble MMPs in blood. This hypothesis was adopted and tested by Korematsu et al., which showed that MMP-9 and MMP-1 were distributed predominantly in granulocytes and platelets were significant risk factors for coronary aneurysms, and correlated with neutrophil count in plasma, and the elevated MMP-1 and MMP-9 levels (16). Our study differs from the study by Korematsu et al. in that we further explored the roles of more MMPs and explored the correlation between MMP levels and clinical parameters of KD patients. The many members of MMP family have quite distinctive structures and functions and therefore it is imperative to elucidate the key MMPs with diagnostic and therapeutic values in clinical management of KD. MMP-2 is known for its role in regulating vascular smooth muscle cell migration, arterial remodeling and many endothelial dysfunction (17). MMP-3 is capable of degrading a wide range of substrates including gelatin 
TABLE 3 | Comparison of MMP-2 and MMP-9 levels among different groups.

\begin{tabular}{|c|c|c|c|c|c|c|c|}
\hline & \multicolumn{3}{|c|}{ Primary group } & \multirow[t]{2}{*}{$p$-value } & \multicolumn{2}{|c|}{ Sub group } & \multirow[t]{2}{*}{$p$-value } \\
\hline & KD & FC & NC & & CAL & Non-CAL & \\
\hline MMP-2 (pg/ml) & $765.47 \pm 346.74^{\star \&}$ & $295.65 \pm 56,32$ & $289.42 \pm 40.07$ & $<0.001$ & $776.43 \pm 359.27$ & $735.64 \pm 321.23$ & 0.014 \\
\hline MMP-9 (pg/ml) & $421.94 \pm 146.38^{\star \&}$ & $101.35 \pm 79.43$ & $111.79 \pm 73.56$ & $<0.001$ & $458.94 \pm 170.31$ & $410.65 \pm 197.24$ & 0.006 \\
\hline
\end{tabular}

MMP-2, 9, metalloproteinases-2, 9; KD, Kawasaki disease; FC, febrile controls; NC, healthy controls; CALs, coronary artery lesions.

" $p$-value of $<0.001$ vs. NC.

${ }^{\&} p$-value of $<0.001$ vs. FC.

TABLE 4 | Changes of MMP-2, and MMP-9 levels before and after treatment.

\begin{tabular}{lcrr}
\hline & KD & Sub group & p-value \\
\cline { 3 - 4 } & & KD-CAL & KD-NonCAL \\
\hline$\Delta$ MMP-2 $(\mathrm{pg} / \mathrm{ml})$ & $409.32 \pm 213.43^{\star \star \star}$ & $412.84 \pm 197.48^{\star \star \star}$ & $404.25 \pm 204.19^{\star \star \star}$ \\
$\Delta \mathrm{MMP}-9(\mathrm{pg} / \mathrm{ml})$ & $05.43 \pm 135.62^{\star \star \star}$ & $210.77 \pm 117.64^{\star \star \star}$ & $186.37 \pm 128.02^{\star \star \star}$ \\
\hline
\end{tabular}

MMP-2, 9, metalloproteinases-2, 9; KD, Kawasaki disease; CALs, coronary artery lesions.

${ }^{* * *} p$-value of $<0.001$ pre-treatment vs. post-treatment.

TABLE 5 | Correlations analysis between MMP-2 and MMP-9 levels and clinical parameters in KD patients.

\begin{tabular}{|c|c|c|c|c|}
\hline & \multicolumn{2}{|c|}{ MMP-2 } & \multicolumn{2}{|c|}{ MMP-9 } \\
\hline & $r$ & $p$ & $r$ & $p$ \\
\hline Fever time & 0.307 & $0.015^{\star}$ & 0.412 & $<0.001^{\star \star \star}$ \\
\hline WBC & 0.312 & $0.003^{\star *}$ & 0.486 & $<0.001^{\star \star \star}$ \\
\hline $\mathrm{RBC}$ & 0.095 & 0.385 & 0.023 & 0.446 \\
\hline Hemoglobin & -0.083 & 0.324 & -0.091 & 0.179 \\
\hline Platelet & 0.107 & 0.337 & 0.121 & 0.285 \\
\hline PCT & 0.309 & $0.011^{*}$ & 0.397 & $0.003^{\star \star}$ \\
\hline CRP & 0.301 & $0.012^{*}$ & 0.472 & $<0.001^{\star * \star}$ \\
\hline ESR & 0.297 & 0.132 & 0.285 & 0.095 \\
\hline IL-6 & 0.325 & $0.007^{\star \star}$ & 0.437 & $<0.001^{\text {*** }}$ \\
\hline MCP-1 & 0.298 & $0.037^{\star}$ & 0.375 & $0.006^{\star \star}$ \\
\hline
\end{tabular}

MMP-2, 9, metalloproteinases-2, 9; KD, Kawasaki disease; WBC, white blood cell counts; $R B C$, red blood cell counts; $C R P, C$-reactive protein; ESR, erythrocyte sedimentation rate; $P C T$, procalcitonin; IL-6, interleukin 6; MCP-1, monocyte chemoattractant protein-1. * $p$-value of $<0.05,{ }^{* *} p$-value of $<0.01,{ }^{* * *} p$-value of $<0.001$.

types I, III, IV and V, and collagen types III, IV, IX and X. Besides, MMP-3 also serves as an activator of other MMPs (18). MMP-9 is responsible for degrading the subendothelial layer mainly composed of collagen, elastin and proteoglycan, which is most closely related to vascular diseases (19). To our best knowledge, most related studies supported the MMP-2 (14) and MMP-9 $(20,21)$ upregulation as a hallmark of KD. Consistent with the retrospective study by Wang et al. (19), which evaluated the predictive role of MMP-9 in evaluating CAL risk in $\mathrm{KD}$ patients, a prospective study like ours would be useful as another confirmation to facilitate the translation of the MMP-9 test in clinics. Sakata et al. reported that only MMP-9 was shown to be upregulated in KD but not MMP-2 (22). We believe this discrepancy could be attributed to the difference in subjects in their study and ours, i.e., more than half of our KD patients had CAL while Sakata et al. included subjects with no coronary sequelae. The subjects in the study by Sakata et al. also received intravenous immunoglobulin therapy, while patients who received treatments were excluded in our study. The finding that MMP-2 and MMP-9 are significantly upregulated in KD qualified the use of those three MMPs as potential biomarkers for diagnosis of KD. Further, the reduction of these MMPs by IVIG treatment justified the measurement of these MMPs in a blood test as strong indicators of therapeutic responses of KD treatment. Our results also suggest that MMP-2 and MMP-2 levels may be used to construct a mathematical model to predict CAL occurrence in KD patients, which is worth pursuing in the future.

To stratify the correlation of upregulation of MMPs with CAL caused by KD, we performed subgroup analysis, which showed that a more prominent upregulation of MMP-2 and MMP-9 was seen in KD patients with CAL, compared to those without CAL. This data indicates the role of MMP-2 and MMP-9 in the pathogenesis of CAL caused by KD, and the value of these MMPs in managing acute KD.

Prior studies have explored the role of MMP upregulation with vascular system abnormalities $(5,23)$. However, due to the complex role of MMPs (24), it is imperative to clarify which MMPs can serve as a therapeutic target of CAL caused by KD. The fact that some clinical parameters of $\mathrm{KD}$ are distinguishable between those with CAL and without CAL, such as fever time, WBC, hemoglobin, etc., while MMP-2 and MMP-9 are remarkably distinctive, suggests that the use of the levels of MMP-2 and MMP-9 for diagnosing KD are of greater clinical value. Previous reports have identified that MMP-2, 9 and 25 upregulation are correlated with abdominal aortic aneurysm (25-27). Which therefore makes them amenable molecules for surveillance and treatment of abdominal lesions. Our data show 
that the clinical potential of MMP-2 and MMP-9 can also be extended to CAL caused by KD. Inspired by the suppression of MMP expression by IVIG treatment, which led to alleviation of the symptoms of $\mathrm{KD}$, new development of therapeutic strategies based on MMPs is desirable; the advances in targeted suppression of MMPs, e.g., by gene therapy $(28,29)$ may achieve a higher efficacy.

We found a significant correlation between the levels of MMPs with some protein markers of $\mathrm{KD}$, including PCT, CRP, IL-6 and MCP-1, which are markers of dysregulated immunological responses. Previously, the link between MMPs and immunological responses has been demonstrated (30). Therefore, the role of MMP suppression by IVIG may also contribute to rebalance of the immune system.

\section{CONCLUSION}

To conclude, here we analyzed the serum levels of three MMPs, i.e., MMP-2 and MMP-9, in KD patient with or without CAL. Healthy and febrile patients were used as controls. We found that the upregulation of the MMPs investigated in our study correlated well with $\mathrm{KD}$ and those with CAL demonstrated higher levels of MMPs than those without CAL, suggesting

\section{REFERENCES}

1. Wang C-L, Wu Y-T, Liu C-A, Kuo H-C, Yang KD. Kawasaki disease: infection, immunity and genetics. Pediatr Infect Dis J. (2005) 24:9981004. doi: 10.1097/01.inf.0000183786.70519.fa

2. Chen KY, Messina N, Germano S, Bonnici R, Freyne B, Cheung M, et al. Innate immune responses following Kawasaki disease and toxic shock syndrome. PLoS ONE. (2018) 13:e0191830. doi: 10.1371/journal.pone.0191830

3. Iemura $M$, Ishii $M$, Sugimura $T$, Akagi $T$, Kato $H$. Long term consequences of regressed coronary aneurysms after Kawasaki disease: vascular wall morphology and function. Heart. (2000) 83:307-11. doi: 10.1136/heart.83.3.307

4. Zhao L, Wang Y, Suo L. Meta-analysis of the risk factors for coronary artery lesion secondary to Kawasaki disease in chinese children. Zhonghua Er Ke Za Zhi. (2011) 49:459-67.

5. Newby AC. Metalloproteinase production from macrophages - a perfect storm leading to atherosclerotic plaque rupture and myocardial infarction. Exp Physiol. (2016) 101:1327-37. doi: 10.1113/EP085567

6. Garlet GP, Cardoso C, Silva T, Ferreira B, Ávila-Campos MJ, Cunha FDQ, et al. Cytokine pattern determines the progression of experimental periodontal disease induced by Actinobacillus actinomycetemcomitans through the modulation of MMPs, RANKL, and their physiological inhibitors. Oral Microbiol Immunol. (2006) 21:12-20. doi: 10.1111/j.1399-302X.2005.00245.x

7. Lehrke M, Greif M, Broedl UC, Lebherz C, Laubender RP, Becker A, et al. MMP-1 serum levels predict coronary atherosclerosis in humans. Cardiovasc Diabetol. (2009) 8:50. doi: 10.1186/1475-2840-8-50

8. Overall CM, López-Otín C. Strategies for MMP inhibition in cancer: innovations for the post-trial era. Nat Rev Cancer. (2002) 2:657. doi: $10.1038 / \mathrm{nrc} 884$

9. Green M, Gough A, Devlin J, Smith J, Astin P, Taylor D, et al. Serum MMP-3 and MMP-1 and progression of joint damage in early rheumatoid arthritis. Rheumatology. (2003) 42:83-8. doi: 10.1093/rheumatology/ keg037

10. Raffetto JD, Khalil RA. Matrix metalloproteinases and their inhibitors in vascular remodeling and vascular disease. Biochem Pharmacol. (2008) 75:34659. doi: 10.1016/j.bcp.2007.07.004 a strong link between MMPs upregulation and pathogenesis of CAL. IVIG treatment also induced a significant reduction of MMP levels. Our study indicated that MMPs are potential biomarkers of KD.

\section{DATA AVAILABILITY STATEMENT}

The raw data supporting the conclusions of this article will be made available by the authors, without undue reservation.

\section{ETHICS STATEMENT}

The studies involving human participants were reviewed and approved by Maternal and Child Health Hospital. The patients/participants provided their written informed consent to participate in this study.

\section{AUTHOR CONTRIBUTIONS}

FT, LM, RZ, LJ, XW, WS, and YJ: data collection and analysis. FT and YJ: study designed and manuscript writing. All authors approved the final submission.

11. Letra A, Ghaneh G, Zhao M, Ray H, Francisconi CF, Garlet GP, et al. MMP7 and TIMP-1, new targets in predicting poor wound healing in apical periodontitis. J Endod. (2013) 39:1141-6. doi: 10.1016/j.joen.2013.06.015

12. Ding X-W, Sun X, Shen X-F, Lu Y, Wang J-Q, Sun Z-R, et al Propofol attenuates TNF- $\alpha$-induced MMP-9 expression in human cerebral microvascular endothelial cells by inhibiting Ca 2+/CAMK II/ERK/NF-кB signaling pathway. Acta Pharmacol Sin. (2019) 40:1303-13. doi: 10.1038/s41401-019-0258-0

13. Zhang Y-H, He Y-E, Xiang R-L, Wu R-Z, Xu Q, Rong X, et al. Effects of $\gamma$ globulin on MMP-2, MMP-9 and TNF- $\alpha$ secretion in human umbilical veins endothelial cells stimulated by serum from children with Kawasaki disease. Chinese J Clin Pharmacol Ther. (2009) 14:586.

14. Gavin PJ, Crawford SE, Shulman ST, Garcia FL, Rowley AH. Systemic arterial expression of matrix metalloproteinases 2 and 9 in acute Kawasaki disease. Arterioscler Thromb Vasc Biol. (2003) 23:576-81. doi: 10.1161/01.ATV.0000065385.47152.FD

15. Mccrindle BW, Li JS, Minich LL, Colan SD, Atz AM, Takahashi $\mathrm{M}$, et al. Coronary artery involvement in children with Kawasaki disease: risk factors from analysis of serial normalized measurements. Circulation. (2007) 116:174-9. doi: 10.1161/CIRCULATIONAHA.107.6 90875

16. Korematsu S, Ohta Y, Tamai N, Takeguchi M, Goto C, Miyahara H, et al. Cell distribution differences of matrix metalloproteinase- 9 and tissue inhibitor of matrix metalloproteinase-1 in patients with Kawasaki disease. Pediatr Infect Dis J. (2012) 31:973-4. doi: 10.1097/INF.0b013e31825ba6b3

17. Belo VA, Parente JM, Tanus-Santos JE, Castro MM. Matrix metalloproteinase (MMP)-2 decreases calponin-1 levels and contributes to arterial remodeling in early hypertension. Biochem Pharmacol. (2016) 118:50-8. doi: 10.1016/j.bcp.2016.08.012

18. Guizani I, Zidi W, Zayani Y, Boudiche S, Hadj-Taieb S, Sanhaji H, et al. Matrix metalloproteinase-3 predicts clinical cardiovascular outcomes in patients with coronary artery disease: a 5 years cohort study. Mol Biol Rep. (2019) 46:4699707. doi: 10.1007/s11033-019-04914-4

19. Wang L, Yang Y, Cui Q, Cui Y, Li Q, Che X, et al. Evaluating the added predictive ability of MMP-9 in serum for Kawasaki disease with coronary artery lesions. J Investig Med. (2020) 69:13-9. doi: 10.1136/jim-2020-001281 
20. Takeshita S, Tokutomi T, Kawase H, Nakatani K, Tsujimoto H, Kawamura Y, et al. Elevated serum levels of matrix metalloproteinase9 (MMP-9) in Kawasaki disease. Clin Exp Immunol. (2001) 125:340-4. doi: 10.1046/j.1365-2249.2001.01608.x

21. Kuo HC, Li SC, Huang LH, Huang YH. Epigenetic hypomethylation and upregulation of matrix metalloproteinase 9 in Kawasaki disease. Oncotarget. (2017) 8:60875-91. doi: 10.18632/oncotarget.19650

22. Sakata K, Hamaoka K, Ozawa S, Niboshi A, Yahata T, Fujii M, et al. Matrix metalloproteinase-9 in vascular lesions and endothelial regulation in Kawasaki disease. Circ J. (2010) 74:1670-5. doi: 10.1253/circj.CJ-09-0980

23. Kadoglou NP, Liapis CD. Matrix metalloproteinases: contribution to pathogenesis, diagnosis, surveillance and treatment of abdominal aortic aneurysms. Curr Med Res Opin. (2004) 20:419-32. doi: 10.1185/030079904125003143

24. Sang QXA. Complex role of matrix metalloproteinases in angiogenesis. Cell Res. (1998) 8:171. doi: 10.1038/cr.1998.17

25. Abdul-Hussien H, Hanemaaijer R, Verheijen JH, Van Bockel JH, Geelkerken RH, Lindeman JH. Doxycycline therapy for abdominal aneurysm: Improved proteolytic balance through reduced neutrophil content. J Vasc Surg. (2009) 49:741-9. doi: 10.1016/j.jvs.2008.09.055

26. Ramella M, Boccafoschi F, Bellofatto K, Follenzi A, Fusaro L, Boldorini R, et al. Endothelial MMP-9 drives the inflammatory response in abdominal aortic aneurysm (AAA). Am J Transl Res. (2017) 9:5485.

27. Jiang H, Sasaki T, Jin E, Kuzuya M, Cheng XW. Inflammatory cells and proteases in abdominal aortic aneurysm and its complications. Curr Drug Targets. (2018) 19:1289-96. doi: 10.2174/13894501196661805311 03458
28. Li M, Zhou X, Zeng X, Wang C, Xu J, Ma D, et al. Folate-targeting redox hyperbranched poly (amido amine) s delivering MMP-9 siRNA for cancer therapy. J Mater Chem B. (2016) 4:547-56. doi: 10.1039/C5TB0 $1964 \mathrm{H}$

29. Wei C, He Z, Kong W. Application of gene therapy in auditory system diseases. STEMedicine. (2020) 1:e17. doi: 10.37175/stemedicine.v1i1.17

30. Goetzl EJ, Banda MJ, Leppert D. Matrix metalloproteinases in immunity. J Immunol. (1996) 156:1-4.

Conflict of Interest: The authors declare that the research was conducted in the absence of any commercial or financial relationships that could be construed as a potential conflict of interest.

Publisher's Note: All claims expressed in this article are solely those of the authors and do not necessarily represent those of their affiliated organizations, or those of the publisher, the editors and the reviewers. Any product that may be evaluated in this article, or claim that may be made by its manufacturer, is not guaranteed or endorsed by the publisher.

Copyright (c) 2022 Tian, Ma, Zhao, Ji, Wang, Sun and Jiang. This is an open-access article distributed under the terms of the Creative Commons Attribution License (CC $B Y)$. The use, distribution or reproduction in other forums is permitted, provided the original author(s) and the copyright owner(s) are credited and that the original publication in this journal is cited, in accordance with accepted academic practice. No use, distribution or reproduction is permitted which does not comply with these terms. 